\title{
Quasi-TEM Analysis of Multilayered, Multiconductor Coplanar Structures with Dielectric and Magnetic Anisotropy Including Substrate Losses
}

\author{
MANUEL HORNO, MEMBER, IEEE, FRANCISCO L. MESA, FRANCISCO MEDINA,
} AND RICARDO MARQUÉS

\begin{abstract}
In this paper, a quasi-TEM analysis of multiconductor planar lines embedded in a layered structure involving lossy iso/ anisotropic electric and/or magnetic materials is achieved. Conditions under which a quasi-TEM assumption is valid are theoretically determined. An efficient spectral-domain analysis is used to determine the complex capacitance, $[C]$ and inductance, $[L]$, matrices characterizing the transmission system. The computation of $[L]$, when media characterized for a fully general permeability tensor are present, is reduced to the computation of an equivalent capacitance matrix. It is also shown that most actual MMIC microstrip-type structures (where semiconductor substrates are present) and possible future applications including lossy magnetic materials can be analyzed by using the simple quasi-TEM model. The validity of the results has been verified by comparison with "full-wave" theoretical and experimental data on microstrip lines on magnetic substrates and slow-wave structures.
\end{abstract}

\section{INTRODUCTION}

$\mathrm{T}$ HE EVOLUTION in microwave systems points at the complete integration of passive and active elements (MMIC). Microstrip-like transmission lines on semiconductor substrates used in these kinds of circuits should be conveniently characterized. The high conductivity of these substrates precludes the use of perturbational techniques in order to take into account the effect of the lossy material on the propagation characteristics. In particular, the important "slow-wave" modes supported by metal-insulator-semiconductor (MIS) configurations must be subjected to more detailed analysis [1]-[3]. The use of magnetic materials in order to improve the behavior of slow-wave lines has been suggested in [4], where the authors develop a qualitative discussion on the subject. ${ }^{1}$

The cross section of the structure considered in this work is shown in Fig. 1, where the elements of the permittivity and permeability tensors of every layer, $[\epsilon]_{i}$

Manuscript received November 15, 1989; revised March 12, 1990. This work was supported by the DGICYT, Spain (Project PB87-0788-CO3-01).

The authors are with the Departamento de Electrónica y Electromagnetismo, Facultad de Fisica, Universidad de Sevilla, 41012 Seville, Spain. IEEE Log Number 9036419.

During the reviewing period, a paper dealing with planar transmission lines with anisotropic magnetic media was published [5].
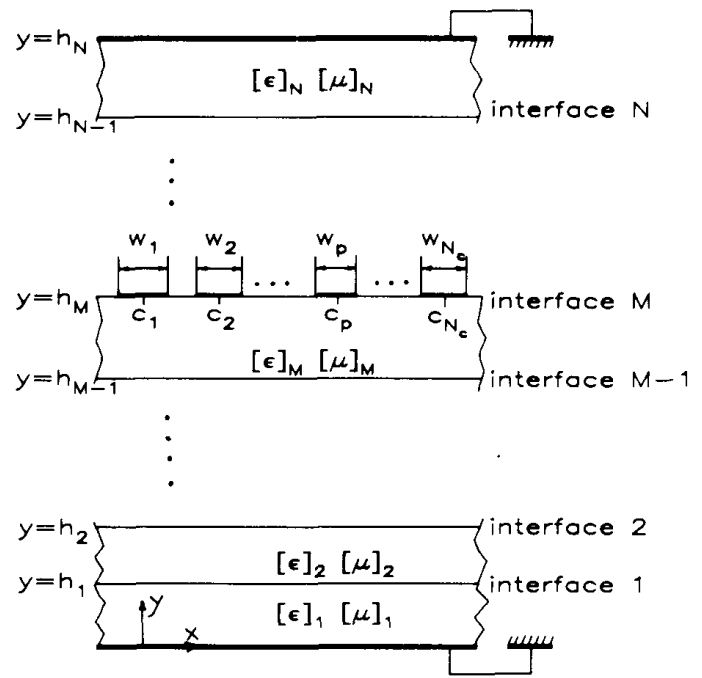

Fig. 1. Transversal section of a multilayer, multiconductor, coplanar structure with dielectric and magnetic anisotropy including losses.

and $[\mu]_{i}$, respectively, are in general complex quantities:

$$
\begin{aligned}
{[\xi]_{i} } & =\xi_{0}\left(\begin{array}{ll}
\xi_{x x}^{i} & \xi_{x y}^{i} \\
\xi_{y x}^{i} & \xi_{y y}^{i}
\end{array}\right) \\
\xi_{\alpha \beta}^{i} & =\left(\xi_{\alpha \beta}^{i}\right)^{\prime}+j\left(\xi_{\alpha \beta}^{i}\right)^{\prime \prime} \\
\xi & =\epsilon \text { or } \mu \\
\alpha \text { or } \beta & =x \text { or } y .
\end{aligned}
$$

The imaginary parts of these coefficients display the effect of substrate losses.

Although most papers dealing with these structures make use of the "full-wave" approach [1], [2], there is a wide range of situations where the "quasi-TEM" assumption is sufficiently correct [3]. The first purpose of the present paper is to analyze the conditions under which 
the quasi-TEM approach can be considered valid, considering the presence of lossy electric or/and magnetic materials. After that, we provide an efficient method to compute the characteristics of multistrip lines embedded in a complex stratified lossy iso/anisotropic medium. For this, we first state the way of obtaining the $[L]$ complex inductance matrix per unit length by solving an equivalent electric problem and working out an equivalent complex capacitance matrix per unit length, $\left[C^{\mathrm{eq}}\right]$. Later we apply the Galerkin method in the spectral domain to obtain $\left[C^{\mathrm{eq}}\right]$ and the complex capacitance matrix of the structure, $[C]$. Propagation constants and impedances are easily computed from these matrices. The imaginary parts of the $[C]$ and $[L]$ matrices will account for the shunt dielectric and series magnetic losses, respectively. A mathematical appendix showing a technique to improve the convergence of the integrals involved has been included. In this way, a very general and efficient algorithm to treat configurations such as those depicted in Fig. 1 is developed, making clear the range of validity of such a description.

\section{Validity of the Quasi-TEM Approximation}

As was stated above, the analysis carried out on the structure in Fig. 1 is based on the quasi-TEM approximation. In [6] and [7], this approximation is justified for lossless substrates. In the context of this paper, it is necessary to know the limitations of the approximation when electric and/or magnetic losses cannot be neglected.

First, in order to avoid confusion in the notation for anisotropic media, let us consider two conductors, $c_{1}$ and $c_{2}$, embedded in an inhomogenous, isotropic, and lossy medium. Both dielectric permittivity, $\hat{\epsilon}(\boldsymbol{r})$, and magnetic permeability, $\hat{\mu}(\boldsymbol{r})$, as well as the complex propagation constant, $\gamma=\beta-j \alpha$, will be complex quantities. A dimensional analysis of Maxwell's equations will allow us to establish the conditions under which the quasi-TEM approach is reasonable. By separating transversal and longitudinal components of fields and operators (subscript $t$ stands for transversal component and $l_{3}$ is the unit vector in the longitudinal direction), Maxwell's equations are written as

$$
\begin{aligned}
\nabla_{t} \times E_{t} & =-j \omega \hat{\mu}(\boldsymbol{r}) H_{z} l_{3} \\
\nabla_{t} E_{z} & =-j \gamma \boldsymbol{E}_{t}-j \omega \hat{\mu}(\boldsymbol{r}) \boldsymbol{l}_{3} \times \boldsymbol{H}_{t} \\
\nabla_{t} \times \boldsymbol{H}_{t} & =j \omega \hat{\boldsymbol{\epsilon}}(\boldsymbol{r}) E_{z} \boldsymbol{l}_{3} \\
\boldsymbol{\nabla}_{t} H_{z} & =j \gamma \boldsymbol{H}_{t}+j \omega \hat{\epsilon}(\boldsymbol{r}) \boldsymbol{l}_{3} \times \boldsymbol{E}_{t} .
\end{aligned}
$$

In order to carry out the dimensional analysis, (2) is integrated along a path from any point $a$ of conductor $c_{1}$ to any point $b$ of conductor $c_{2}$, and (4) is integrated along a path $C$ surrounding a conductor, namely

$$
\begin{aligned}
& 0=-j \gamma \int_{a}^{b} \boldsymbol{E}_{t} \cdot d \boldsymbol{l}+j \omega \int_{a}^{b} \hat{\mu}(\boldsymbol{r}) \boldsymbol{H}_{t} \cdot\left(\boldsymbol{l}_{3} \times d \boldsymbol{l}\right) \\
& 0=-j \gamma \oint_{C} \boldsymbol{H}_{t} \cdot d \boldsymbol{t}-j \omega \oint_{C} \hat{\boldsymbol{\epsilon}}(\boldsymbol{r}) \boldsymbol{E}_{t} \cdot\left(\boldsymbol{l}_{3} \times d \boldsymbol{t}\right) .
\end{aligned}
$$

For our purpose, we consider

$$
\begin{gathered}
\int_{a}^{b} \boldsymbol{E}_{\boldsymbol{t}} \cdot d \boldsymbol{l} \sim\left\langle\left\|\boldsymbol{E}_{t}\right\|\right\rangle d \\
\int_{a}^{b} \hat{\mu}(\boldsymbol{r}) \boldsymbol{H}_{t} \cdot\left(\boldsymbol{l}_{3} \times d \boldsymbol{l}\right) \sim\langle|\hat{\mu}(\boldsymbol{r})|\rangle\left\langle\left\|\boldsymbol{H}_{t}\right\|\right\rangle d \\
\oint \boldsymbol{H}_{t} d \boldsymbol{t} \sim\left\langle\left\|\boldsymbol{H}_{t}\right\|\right\rangle d \\
\oint \hat{\epsilon}(\boldsymbol{r}) \boldsymbol{E}_{t} \cdot\left(\boldsymbol{l}_{3} \times d \boldsymbol{t}\right) \sim\langle|\hat{\boldsymbol{\epsilon}}(\boldsymbol{r})|\rangle\left\langle\left\|\boldsymbol{E}_{t}\right\|\right\rangle d
\end{gathered}
$$

where $x \sim y$ denotes that the order of magnitude of quantities $x$ and $y$ is the same, $\langle\cdot\rangle$ denotes average value, $|\cdot|$ denotes modulus, $\|\cdot\|$ denotes vector norm, and $d$ is the transversal characteristic dimension of the line.

Combing (5) and (6) and taking into account (7)-(10), we obtain

$$
\left\langle\left|E_{z}\right|\right\rangle \sim|\gamma| d\left\langle\left\|E_{t}\right\|\right\rangle
$$

where

$$
|\gamma| \sim \omega \sqrt{\langle|\hat{\mu}(\boldsymbol{r})|\rangle\langle\hat{\epsilon}(\boldsymbol{r}) \mid\rangle} .
$$

Therefore, we can conclude that the range of validity of the quasi-TEM approximation, $\left\langle\left|E_{z}\right|\right\rangle /\left\langle\left\|E_{t}\right\|\right\rangle \ll 1$, is determined by

$$
d \ll \frac{1}{\omega \sqrt{\langle|\hat{\mu}(\boldsymbol{r})|\rangle\langle\hat{\hat{\epsilon}(\boldsymbol{r})|\rangle}}} .
$$

Condition (13) is easily extended to the anisotropic case. For it, we have to substitute $\hat{\mu}(\boldsymbol{r})$ or $\hat{\epsilon}(\boldsymbol{r})$ in this expression for the largest element in the tensor permeability or permittivity.

Next, we will consider separately the effects of dielectric and magnetic losses on condition (13).

\section{A. Dielectric Lossy Medium}

In this case (13) becomes

$$
d \ll \frac{1}{\omega\left[\langle\mu(\boldsymbol{r})\rangle\langle\epsilon(\boldsymbol{r})\rangle \sqrt{1+\frac{\langle\sigma(\boldsymbol{r})\rangle^{2}}{\omega^{2}\langle\epsilon(\boldsymbol{r})\rangle^{2}}}\right]^{1 / 2}} .
$$

Two typical limit situations often arise in practice. The first is $\langle\sigma(r)\rangle^{2} \ll \omega^{2}\langle\epsilon(r)\rangle^{2}$. In this case (14) becomes

$$
d \ll \frac{1}{\omega \sqrt{\langle\mu(\boldsymbol{r})\rangle\langle\epsilon(\boldsymbol{r})\rangle}} .
$$

Equation (15) is the usual expression for the case of lossless medium. The second limit situation is $\langle\sigma(r)\rangle^{2} \gg$ $\omega^{2}\langle\epsilon(r)\rangle^{2}$. In this case,

$$
d \ll \frac{1}{\sqrt{\omega\langle\mu(\boldsymbol{r})\rangle\langle\sigma(\boldsymbol{r})\rangle}} .
$$




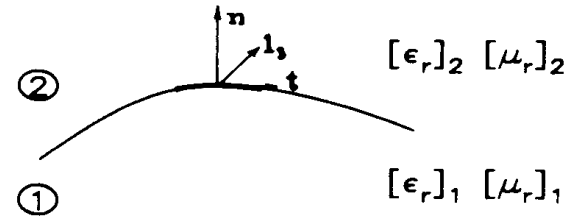

(a)

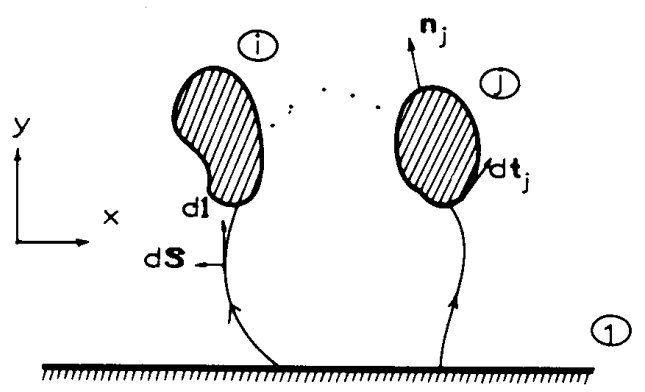

(b)

Fig. 2. (a) Transversal section of a general interface. (b) Transversal section of a multiconductor line.

Thus it is obtained that $d \ll\langle\delta(\boldsymbol{r})\rangle$, where $\delta$ is the skin depth.

The above situations are for a dielectric with small losses and a semiconductor substrate respectively. In the first case, condition (15) shows that the quasi-TEM model is valid when the transverse dimensions are much smaller than the wavelength, such as in the lossless case. When the substrate is a semiconductor, quasi-TEM propagation is in essence allowed if the skin effect can be neglected.

\section{B. Magnetic Lossy Medium}

If the medium is a demagnetized homogeneous and lossy ferrite which is characterized by a scalar permeability (see, for instance, [8] for an expression of this quantity), (13) becomes velocity of light [6], via

$$
[L]=\frac{1}{c^{2}}\left[C_{0}\right]^{-1}
$$

As is well known, if magnetic media are involved, this simple relation does not hold. It is then necessary to solve a magnetostatic problem for computing the $[L]$ matrix. However, [9] and [10] show the analogy between this magnetic problem and other equivalent dielectric problems when the magnetic medium is isotropic. Therefore, these works are not strictly applicable to the treatment of important cases, such as partially magnetized or saturated ferrites. In this section, the equivalence is extended to magnetic materials characterized by a general permeability tensor.

\section{A. Isomorphism Between Magnetic and Dielectric Problems}

Let us consider a general interface between two media (see Fig. 2(a)), characterized by the relative permeability and permittivity tensors $\left[\mu_{r}\right]_{i}$ and $\left[\epsilon_{r}\right]_{i}(i=1,2)$, respectively, which can be complex quantities. Electric and magnetic fields and potentials obey the following partial differential equations and boundary conditions:

1) Electric Field:

$$
\nabla_{t} \cdot\left(\left[\epsilon_{r}\right]_{i} \cdot \nabla_{t} \phi_{e, i}\right)=0 \quad(i=1,2)
$$

$\phi_{e}$ being the electric potential. For the dielectric interface,

$$
\begin{gathered}
E_{t 1}=E_{t 2} \Rightarrow\left(l_{3} \times n\right) \cdot \nabla_{t} \phi_{e 1}=\left(l_{3} \times n\right) \cdot \nabla_{t} \phi_{e 2} \\
D_{n 1}=D_{n 2} \Rightarrow\left(\left[\epsilon_{r}\right]_{1} \cdot \nabla_{t} \phi_{e 1}\right) \cdot n=\left(\left[\epsilon_{r}\right]_{2} \cdot \nabla_{t} \phi_{e 2}\right) \cdot n
\end{gathered}
$$

\section{1}

$$
d \ll \frac{1}{\omega \sqrt{\mu_{0} \epsilon} \sqrt{\left\{\frac{2}{3}\left[1-\left(\frac{\gamma 4 \pi M_{s}}{\omega}\right)^{2}\right]^{1 / 2}+\frac{1}{3}\right\}^{2}+A^{2}\left(\frac{\gamma 4 \pi M_{s}}{\omega}\right)^{2 N}}}
$$

where $\gamma$ is the gyromagnetic ratio, $4 \pi M_{s}$ is the saturation magnetization, and $A$ and $N$ are adjustable parameters in the imaginary part of scalar permeability.

As was expected, the accuracy of the quasi-TEM model decreases when frequency increases for both kinds of media. Nevertheless, in MMIC technology the value of the transversal distances and the range of useful frequencies justify the use of the quasi-TEM approximation for most practical cases.

\section{Equivalence Between Magnetic and Dielectric Media}

In the range of validity of the quasi-TEM assumption, if the medium is nonmagnetic, the inductance matrix is related to $\left[C_{0}\right]$, the vacuum capacitance matrix, and $c$, the
For the conductor interface,

$$
\begin{gathered}
E_{t 1}=0 \Rightarrow\left(l_{3} \times n\right) \cdot \nabla_{t} \phi_{e 1} \\
D_{n 1}=-\rho_{s} \Rightarrow\left(\left[\epsilon_{r}\right]_{1} \cdot \nabla_{t} \phi_{e 1}\right) \cdot n=-\frac{\rho_{s}}{\epsilon_{0}} .
\end{gathered}
$$

2) Magnetic Field: Since field $\boldsymbol{B}$ has only transversal components, $\boldsymbol{B}$ can be written as $\boldsymbol{B}=-\boldsymbol{l}_{3} \times \nabla_{t} A_{z}$, where $A_{z}$ is the $z$ component of the vector magnetic potential. With $[\eta]_{i}=\left[\mu_{r}\right]_{i}^{-1}$, the equation for this component is

$$
\nabla_{t} \times\left[[\eta]_{i} \cdot\left(l_{3} \times \nabla_{t} A_{z, i}\right)\right]=0 \quad(i=1,2)
$$


By means of mathematical manipulation, the following identity can be proved:

$$
[\eta]_{i} \cdot\left(l_{3} \times \nabla_{t} A_{z, i}\right)=\frac{1}{\operatorname{det}\left[\mu_{r}\right]_{i}} l_{3} \times\left(\left[\mu_{r}\right]_{i}^{t} \cdot \nabla_{t} A_{z, i}\right) \text {. }
$$

Using (25), (24) can be rewritten as

$$
\nabla_{t} \cdot\left[\frac{\left[\mu_{r}\right]_{i}^{t}}{\operatorname{det}\left[\mu_{r}\right]_{i}} \cdot \nabla_{t} A_{z, i}\right]=0 \quad(i=1,2) .
$$

Boundary conditions for the magnetic field are, for the magnetic interface,

$$
\begin{aligned}
B_{n 1}=B_{n 2} \Rightarrow & \left(l_{3} \times n\right) \cdot \nabla_{t} A_{z 1}=\left(l_{3} \times n\right) \cdot \nabla_{t} A_{z 2} \\
H_{t 1}=H_{t 2} \Rightarrow & {\left[[\eta]_{1} \cdot\left(l_{3} \times \nabla_{t} A_{z 1}\right)\right] \cdot t } \\
& =\left[[\eta]_{2} \cdot\left(l_{3} \times \nabla_{t} A_{z 2}\right)\right] \cdot t .
\end{aligned}
$$

Making use of (25), (28) becomes

$$
\boldsymbol{n} \cdot\left[\frac{\left[\mu_{r}\right]_{1}^{t}}{\operatorname{det}\left[\mu_{r}\right]_{1}} \cdot \nabla_{t} A_{z 1}\right]=\boldsymbol{n} \cdot\left[\frac{\left[\mu_{r}\right]_{2}^{t}}{\operatorname{det}\left[\mu_{r}\right]_{2}} \cdot \nabla_{t} A_{z 2}\right] \text {. }
$$

For the conductor interface,

$$
\begin{aligned}
B_{n 1}=0 & \Rightarrow\left(l_{3} \times n\right) \cdot \nabla_{t} A_{z 1}=0 \\
n \times H_{1}=j_{s} & \Rightarrow n \times\left[[\eta]_{1} \cdot\left(l_{3} \times \nabla_{t} A_{z 1}\right)\right]=-\mu_{0} j_{s} .
\end{aligned}
$$

We can note that $(31)$ is

$$
n \cdot\left[\frac{\left[\mu_{r}\right]_{1}^{t}}{\operatorname{det}\left[\mu_{r}\right]_{1}} \cdot \nabla_{t} A_{z 1}\right]=-\mu_{0} j_{z} .
$$

Comparing (19)-(23) with (26), (27), (29), (30), and (32), we can note that these sets of equations are identical. So, we can state an isomorphism between $\phi_{e}$ and $A_{z}$ by means of the relation

$$
\left[\epsilon_{r}\right]_{i}=\frac{1}{\operatorname{det}\left[\mu_{r}\right]_{i}}\left[\mu_{r}\right]_{i}^{t} \quad(i=1,2) .
$$

This isomorphism allows us to determine the magnetic field, when the magnetic medium is characterized by a general permeability tensor, by solving an equivalent electric problem.

\section{B. Computation of [L]}

Let us consider the structure in Fig. 2(b). The complex inductance matrix coefficients are defined as

$$
L_{i j}=\left.\frac{\Phi_{m i}}{I_{j}}\right|_{\forall I_{i \neq j}=0} .
$$

$\Phi_{m i}$ is the magnetic flux per unit length associated with conductor $i$, which can be expressed as

$$
\Phi_{m i}=\int_{1}^{i} \nabla_{t} A_{z i} \cdot d l=A_{z i} .
$$

Conductor 1 has been considered the zero magnetic potential level.

We can rewrite (34) as

$$
L_{i j}=-\left.\frac{A_{z i}}{\frac{1}{\mu_{0}} \oint_{j}\left(n_{j} \cdot \frac{\left[\mu_{r}\right]^{t}}{\operatorname{det}\left[\mu_{r}\right]} \cdot \nabla_{t} A_{z j}\right) d t_{j}}\right|_{\forall I_{i \neq j}=0}
$$

where $\oint_{j}$ denotes integration around the $j$ th conductor boundary. On the other hand, if the $[P]$ matrix is defined as the inverse of the complex capacitance matrix per unit length, $[P]=[C]^{-1}$, we have

$$
P_{i j}=\left.\frac{\phi_{e i}}{q_{j}}\right|_{\forall q_{l \neq j}=0} .
$$

These coefficients can be written as

$$
P_{i j}=-\left.\frac{\phi_{e i}}{\epsilon_{0} \oint_{j}\left(n_{j} \cdot\left[\epsilon_{r}\right] \cdot \nabla_{t} \phi_{e j}\right) d t_{j}}\right|_{\forall q_{l \neq j}=0} .
$$

In subsection III-A it was proved that the magnetic problem can be reduced to an electric equivalent one simply by using the equivalent permittivity given by (33). So, in order to compute $[L]$ and taking into account the form of (36) and (38), we can conclude that $L_{i j}=\epsilon_{0} \mu_{0} P_{i j}^{\text {eq }}$, where $P_{i j}^{\text {eq }}$ is an element of the matrix $\left[P^{\text {eq }}\right]=\left[C^{\text {eq }}\right]^{-1}$, $\left[C^{\mathrm{eq}}\right]$ being the complex capacitance matrix per unit length when the permittivity tensors are given by (33). We can finally write

$$
[L]=\epsilon_{0} \mu_{0}\left[C^{\mathrm{eq}}\right]^{-1} .
$$

Therefore, from the above expression, we can say that the calculation of the inductance matrix can be achieved by computing an equivalent capacitance matrix.

\section{Computation of the Complex Capacitance Matrices}

Under the quasi-TEM approximation, the structure of Fig. 1 is fully characterized by the complex capacitance and inductance matrices per unit length, $[C]$ and $[L]$. The physical meaning of the real and imaginary parts of the [C] matrix is discussed in [11]. Similarly, the real part of $[L]$ is the usual inductance matrix, and the imaginary part is a series resistance matrix related to the magnetic losses. Nevertheless, as stated above, the evaluation of the $[L]$ matrix is reduced to the determination of an equivalent capacitance matrix [ $\left.C^{\mathrm{eq}}\right]$. In consequence, the analysis to determine the characteristic parameters of the line is based entirely upon the calculation of complex capacitance matrices. Therefore we will be interested in the complex capacitance matrix per unit length of a structure such as the one shown in Fig. 1, but without magnetic substrates (since the magnetic part of the problem is replaced by an electric equivalent problem). This matrix relates the complex charge vector $Q$ to the voltage vector $V$ as follows: $Q=[C] \cdot V$.

If all the elements of the voltage vector are set to zero except the $j$ th, which is set to unity, then the $j$ th column of the capacitance matrix is equal to the charge vector, $Q_{p}^{j}=C_{p j}\left(p, j=1, \cdots, N_{c}\right.$; the superscript $j$ indicates which conductor is excited). Therefore, the evaluation of $[C]$ is the evaluation of $N_{c}$ charge vectors corresponding to different excitations. To find the charge distribution on the $M$ th interface and obtain these charge vectors $Q^{j}$, we 
have to solve the following operator equation:

$$
\begin{aligned}
\mathscr{G} \rho & =\phi \\
\rho & =0 \quad \text { outside the surface of the strips }
\end{aligned}
$$

where $\rho=\rho(x)$ and $\phi=\phi(x)$ are, respectively, the charge distribution and the potential on the $M$ th interface (the potential is zero except in the excited strip, which is set to unity), and $\mathscr{G}_{\rho}=\int_{-\infty}^{\infty} G\left(x, x^{\prime}\right) \rho\left(x^{\prime}\right) d x^{\prime}, G\left(x, x^{\prime}\right)$ being the Green's function for $y=h_{M}$.

The Galerkin method is used to solve the above integral equation. If we consider

$$
\rho(x)=\sum_{p=1}^{N_{c}} \rho_{p}(x)
$$

where

$$
\rho_{p}(x) \neq 0 \quad \text { if } \quad c_{p}-\frac{w_{p}}{2} \leqslant x \leqslant c_{p}+\frac{w_{p}}{2}
$$

and $\rho_{\rho}(x)$ is expanded in the finite base of functions $\Psi_{p}=\left\{\rho_{p 0}, \rho_{p 1}, \cdots, \rho_{p N_{f}}\right\}$, then

$$
\rho(x)=\sum_{p=1}^{N_{c}} \sum_{n=0}^{N_{f}} a_{p n}^{j} \rho_{p n}(x) .
$$

(Subscript $p$ or $q$ will refer to the strip and subscript $n$ or $m$ to the basis function.)

Carrying out the Galerkin method, we find the following system of linear equations: $\boldsymbol{b}^{j}=[\Gamma] \boldsymbol{a}^{j}$, where $b_{q m}^{j}=$ $\left(\rho_{q m}, \phi_{j}\right)$ are the elements of the vector $b^{j}$ and $\Gamma_{q m p n}=$ $\left(\rho_{q m}, \mathscr{G} \rho_{p n}\right)$ the elements of the $[\Gamma]$ matrix $(q, p=$ $1, \cdots, N_{c} ; m, n=0, \cdots, N_{f}$ ). The [ $\left.\Gamma\right]$ matrix does not depend on the excitation of the strips. According to the notation used,

$$
C_{i j}=Q_{i}^{j}=\sum_{p=0}^{N_{f}} a_{p i}^{j}\left(\rho_{p i}, \phi_{i}\right)=\left(b^{j}\right)^{t}\left([\Gamma]^{-1}\right)^{t}\left(b^{i}\right) .
$$

This equation is a general expression for the complex capacitance coefficients in terms of the complex Green's function and the different basis functions. If the following well-known basis functions are chosen:

$$
\rho_{p n}(x)=\frac{T_{n}\left(\frac{x-c_{p}}{w_{p} / 2}\right)}{\sqrt{1-\left(\frac{x-c_{p}}{w_{p} / 2}\right)^{2}}}
$$

( $T_{n}$ being the Chebyshev polynomials) and the Parseval identity is used, we will have

$$
b_{q m}^{j}=\delta_{q j} \delta_{m 0} \frac{w_{q}}{2} \pi
$$

and

$$
\begin{aligned}
\Gamma_{q m p n}= & (-j)^{n} j^{m} \pi^{2} \frac{w_{p}}{2} \frac{w_{q}}{2} \\
& \cdot \int_{-\infty}^{\infty} J_{n}\left(\alpha \frac{w_{p}}{2}\right) \tilde{G}(\alpha) J_{m}\left(\alpha \frac{w_{q}}{2}\right) e^{j \alpha\left(c_{q}-c_{p}\right)} d \alpha
\end{aligned}
$$

where $J_{k}(\cdot)$ stands for the Bessel function of order $k$. In order to find the complex Green's function in the spectral domain, $\tilde{G}(\alpha)$, the algorithms developed in [12] have been slightly modified to include the effect of a fully general permittivity tensor (see Appendix I).

At this point, it is very important to consider the numerical efficiency in computing the $\Gamma_{q m p n}$ parameters. If a direct numerical integration is tried (for instance, the Simpson or Romberg method), the slow convergence and the oscillations of the integrand in (45) make the computation time, in standard computers, too long to become practical. Therefore, it is necessary to apply some numerical integration scheme which provides a fast computation. In this paper, an appropriate asymptotic treatment joined with residue calculus techniques (see Appendix II) has allowed us to reduce the computation time. We have typically obtained a ratio between the time employed by direct integration and by these schemes of about 50 .

\section{Results}

In this section, before generating reliable data, we are going to check the efficiency of the method reported in this paper. First, since our analysis makes use of a numerical scheme, we must be sure that the computed values converge when the number of basis functions increases. Second, we need to verify that these values are correct. To ensure the convergence of the numerical method, we will study the influence of the number of basis functions on final numerical data for a variety of significant structures. The validity of the results will be checked by comparison with experimental and theoretical (full-wave) data obtained by other authors. Once all this has been done, we can use our programs with confidence to study several structures with semiconductor and magnetic substrates.

\section{A. Convergence Analysis}

In order to study the convergence of the method, a variety of convergence patterns similar to the ones shown in Fig. 3 have been generated. The relative error is computed taking as reference value the result obtained with a large number of basis functions $\left(N_{f}=7\right)$. For practical dimensions we have concluded that no more than three basis functions are needed to obtain very accurate results. Worst cases always correspond to offdiagonal elements of the capacitance matrices, and even for these elements errors are below 3\%. The larger the absolute values, the smaller the relative errors; therefore, diagonal elements are always computed with an error of less than $1 \%$. On the other hand, we have found that odd-order functions are required to take into account strongly coupled structures, and even-order ones are more significant for large $W / h_{2}$ ratios (for instance in Fig. 3).

\section{B. Numerical Results and Discussion}

We now compare our values with experimental data, when these are available, and with theoretical ones obtained by full-wave analysis [2], [13]. Our purpose is to 
show how nowadays in most interesting practical cases of MMIC technology, a full-wave analysis is not necessary to obtain accurate values. The full-wave analysis is much more involved than the quasi-TEM one, requiring more computer capability. With the asymptotic behavior we have introduced, it is possible to use a PC, and the scheme reported here could be suitable for CAD purposes. Nevertheless, a rigorous full-wave analysis must be carried out in order to establish the frequency behavior when quasi-TEM assumptions are not valid. Next, we present examples of particular structures. First, microstrip on demagnetized lossless ferrite substrates is analyzed in Fig. 4. We can see that in proximity to the resonance frequency, the quasi-TEM approximation is not yet valid. This fact was expected, because around this frequency condition (13) is not fulfilled. For the rest of the frequencies, the agreement is good. In Fig. 5 we represent the cross section of a coupled slow-wave MIS configuration. For this structure, we plot the slow-wave factor and the attenuation constant in Fig. 6. In this figure, some experimental results corresponding to the single strip case [2] have been included for comparison purposes. Even- and odd-mode characteristics tend to these results when the strips are weakly coupled $(s \mapsto \infty)$. The agreement with both analytical full-wave and experimental data is very good in the whole frequency range considered.

Second, we are interested in the application of our programs to slow-wave ferromagnetic microstrip lines such as those discussed in [4] (FM and FMS). In [4], these structures are studied by means of a qualitative parallelplate waveguide model. Since the dimensions and characteristics of the structures analyzed in that paper obey condition (13), we can perform a more accurate study by using the quasi-TEM approximation. Fig. 7 shows the slow-wave factor and the attenuation constant for microstrip lines in which the lower layer in Fig. 5 is considered to be either a demagnetized ferromagnetic substrate (FM) or a demagnetized ferromagnetic semiconductor substrate (FMS). We have chosen several values of the constant $A$ in (17) (the larger the value of $A$, the bigger the losses in the ferromagnetic substrate) to show how the magnetic losses affect the above features. Our results exhibit the same qualitative shape as those in [4], but we can notice that for usual values of $A$ (in [8], $A \sim 10^{-2}$ ), these structures do not offer the important slow-wave effects reported in [4]. Magnetic losses should be much greater than the actual ones to obtain outstanding slowwave behavior. Also, we have computed the slow-wave factor and the attenuation constant for three different microstrip lines: MIS, demagnetized FMS, and saturated FMS. (Expressions for the Polder tensor when the substrate is magnetically saturated parallel to the $z$ axis can be found in [14].) Slight differences between the curves corresponding to the magnetic structures and the MIS one have been found for most geometries. In Fig. 8, this can be checked for a particular geometric configuration. We can note how the shape of the curves is due mainly to the conductivity of the substrate. Therefore, the mere presence of magnetic losses in the substrates has little influence on the improvement of slow-wave effects with respect to the presence of electric ones. In proximity to the resonance frequency $(0.6 \mathrm{GHz}$ for the demagnetized FMS and $6 \mathrm{GHz}$ for the saturated FMS), the permeability tensor elements have higher values. This leads to the discrepancies observed in this range of frequencies.

\section{Conclusions}

A short review of the most relevant points developed in this paper follows.

- A qualitative theoretical justification of the quasiTEM model has been achieved. This concerns the application of this model to the most practical MMIC transmission lines.

- The computation of the complex inductance matrix per unit length, for structures characterized by a general permeability tensor, is reduced to the computation of an equivalent complex capacitance matrix.

- A simple and numerically efficient algorithm to compute the complex capacitance matrix of very general microstrip-like configurations has been developed. This algorithm could be used as a CAD tool, since an appropriate numerical treatment makes it possible to achieve tolerable CPU times.

- The efficiency of the algorithm has been demonstrated by the agreement with experimental data and with theoretical results obtained by means of much more involved full-wave computations. Slow-wave structures such as MIS, FM, and FMS configurations have been successfully analyzed with this algorithm.

\section{APPENDix I}

Following [12], the Green's function in the spectral domain for the structure shown in Fig. 1 is

$$
\frac{1}{\tilde{G}(\alpha)}=H_{M}^{i}(\alpha)+H_{M}^{s}(\alpha)-\tilde{g}_{M M}(\alpha)
$$

where

$$
\begin{aligned}
H_{j}^{i}(\alpha) & =\tilde{g}_{j j}(\alpha)-\frac{\tilde{g}_{j, j-1}(\alpha) \tilde{g}_{j-1, j}(\alpha)}{H_{j-1}^{i}(\alpha)} \\
j & =2, \cdots, M \\
H_{1}^{i}(\alpha) & =\tilde{g}_{11}(\alpha)
\end{aligned}
$$

express the contribution of the layers below the interface $M$, and

$$
\begin{aligned}
H_{j}^{s}(\alpha) & =\tilde{g}_{j j}(\alpha)-\frac{\tilde{g}_{j, j+1}(\alpha) \bar{g}_{j+1, j}(\alpha)}{H_{j+1}^{s}(\alpha)} \\
j & =N-2, \cdots, M \\
H_{N-1}^{s}(\alpha) & =\tilde{g}_{N-1, N-1}(\alpha)
\end{aligned}
$$

express the contribution of the layers above the interface $M$.

The meanings of the functions which appear above are explained in [15]. The generality of the permittivity ten- 


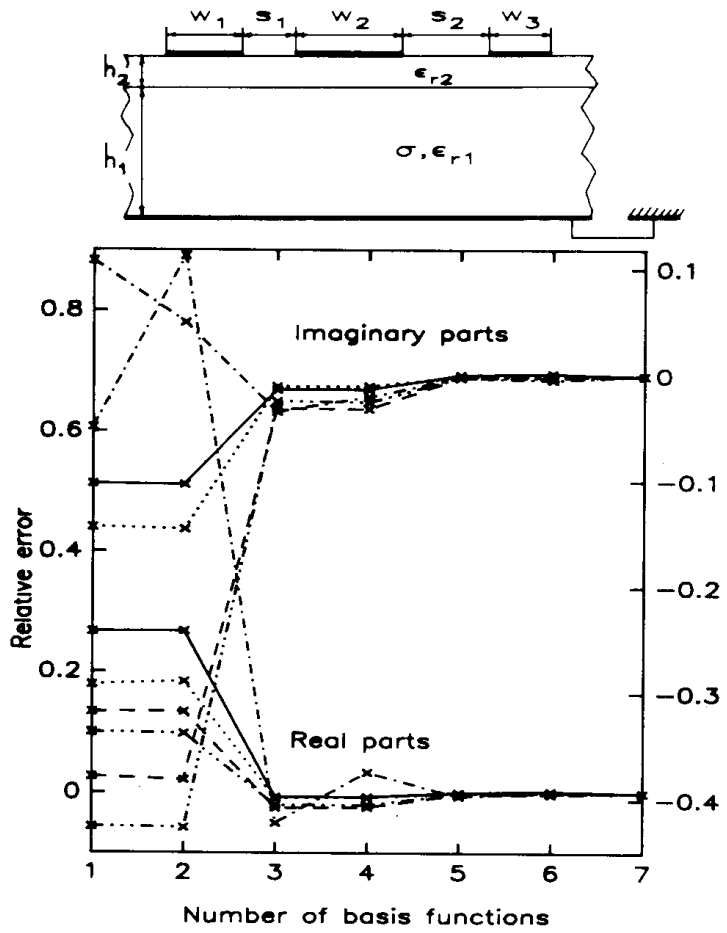

Fig. 3. Relative errors in $1 \%$ of real and imaginary parts of capacitance elements. Freq $=1 \mathrm{GHz} . h_{1}=250 \mu \mathrm{m} . h_{2}=1 \mu \mathrm{m} . w_{1}=160$ $\mu \mathrm{m} . w_{2}=100 \mu \mathrm{m} . w_{3}=120 \mu \mathrm{m} . s_{1}=s_{2}=190 \mu \mathrm{m} . \epsilon_{r 1}=12, \epsilon_{r 2}=4$, $\sigma=8(\Omega \cdot \mathrm{mm})^{-1} \cdot(\longrightarrow) C_{11},(-\cdots--) C_{12},(\ldots \cdot \ldots \cdot \ldots \cdot-\ldots)$ $C_{13},(\cdots \cdots \cdots \cdots \cdots) C_{22},(-\cdots, \cdots, \cdots,) C_{23}$.

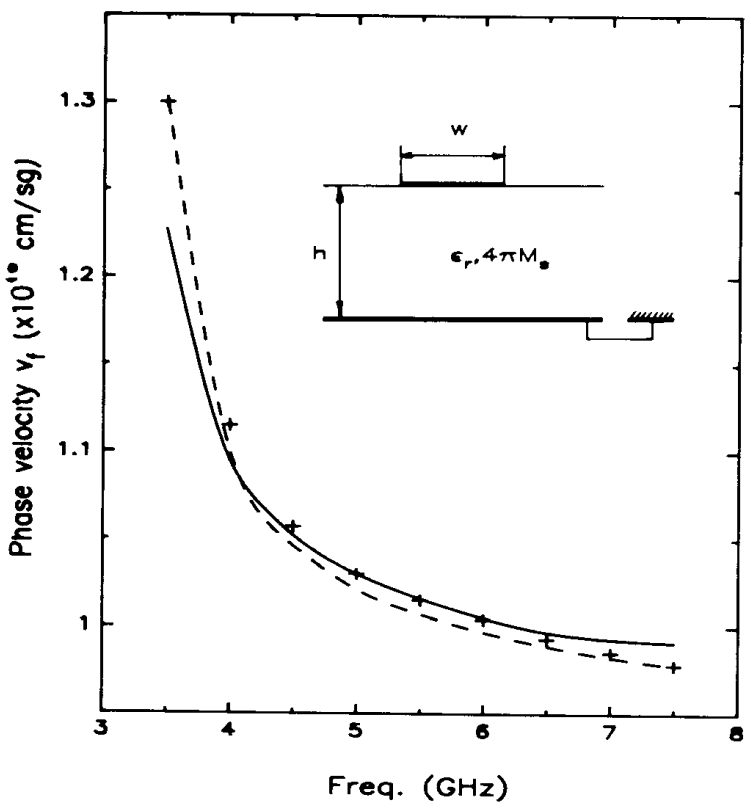

Fig. 4. Phase velocity for a microstrip over ferrite demagnetized lossless substrate G-1001. $w / h=0.431,4 \pi M_{s}=1210$ G. $\epsilon_{r}=15.5$. (-_ Present method, $(---)$ [13], $(+++)$ experimental values.

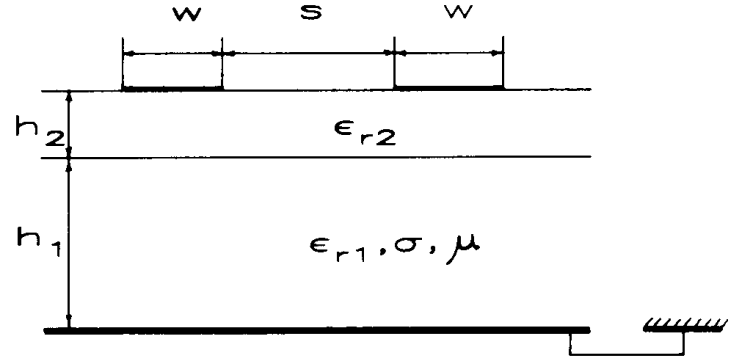

Fig. 5. Transversal section of a coupled microstrip slow-wave configuration (symmetric two conductors). If lower layer substrate is a semiconductor, MIS; if ferromagnetic, FM; if a semiconductor ferromagnetic, FMS
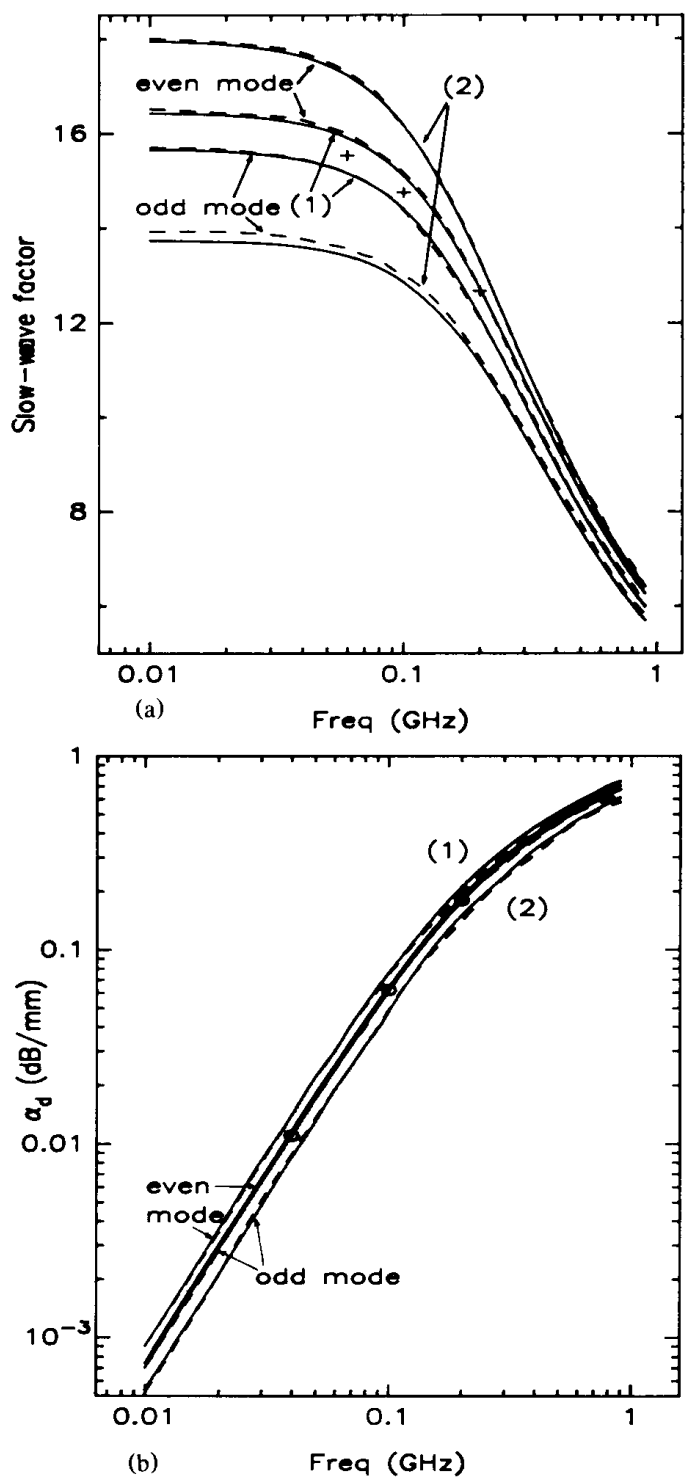

Fig. 6. (a) Slow-wave factor and (b) attenuation constant for the coupled microstrip MIS configuration shown in Fig. 5, with $w=160 \mu \mathrm{m}$, $h_{1}=250 \mu \mathrm{m}, h_{2}=1 \mu \mathrm{m}, \sigma=5 \cdot 10^{-3}(\Omega \cdot \mathrm{mm})^{-1}, \epsilon_{r 1}=\epsilon_{r 2}=12, s=$ $800 \mu \mathrm{m}$ for (1) and $s=160 \mu \mathrm{m}$ for (2). (—) Present method, $(---)[2],(+++)$ or $(\circ \circ \circ)$ experimental values. 

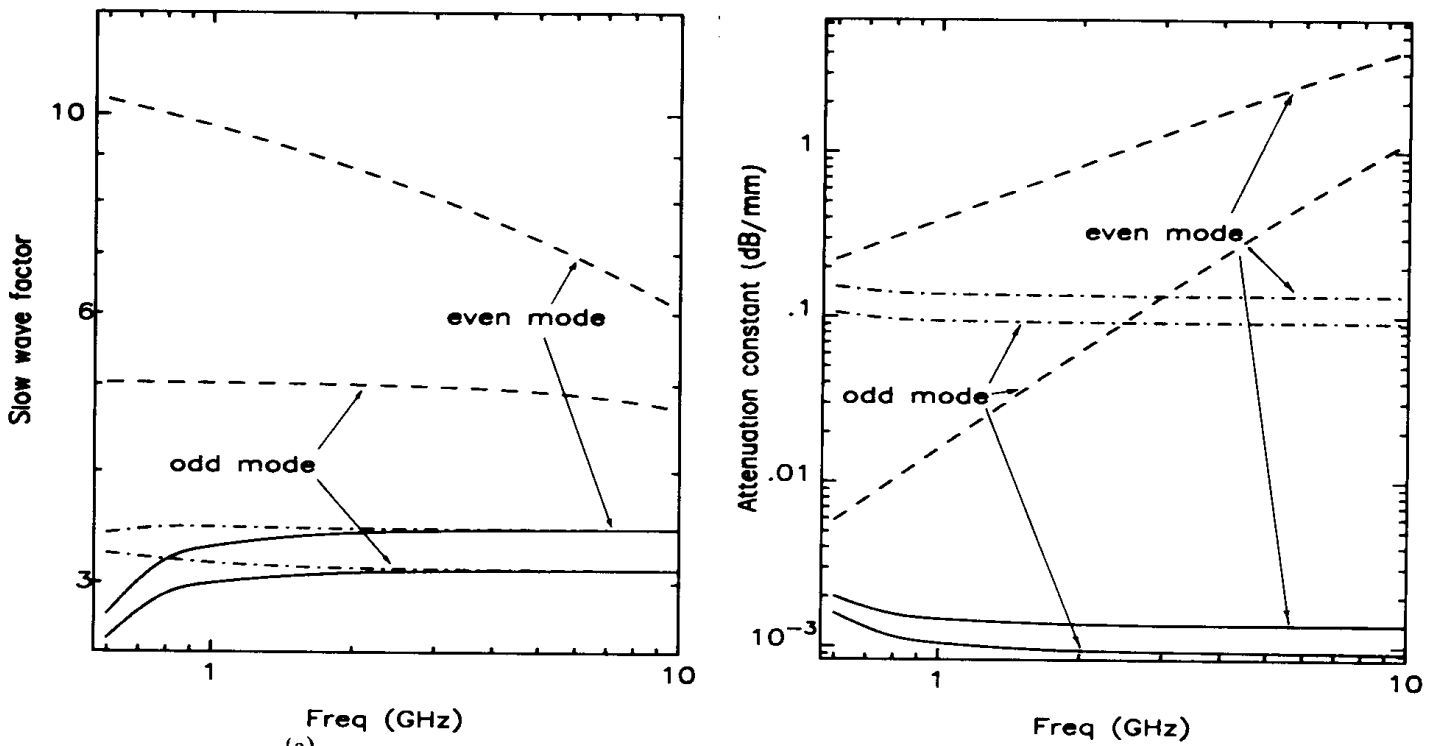

(a)
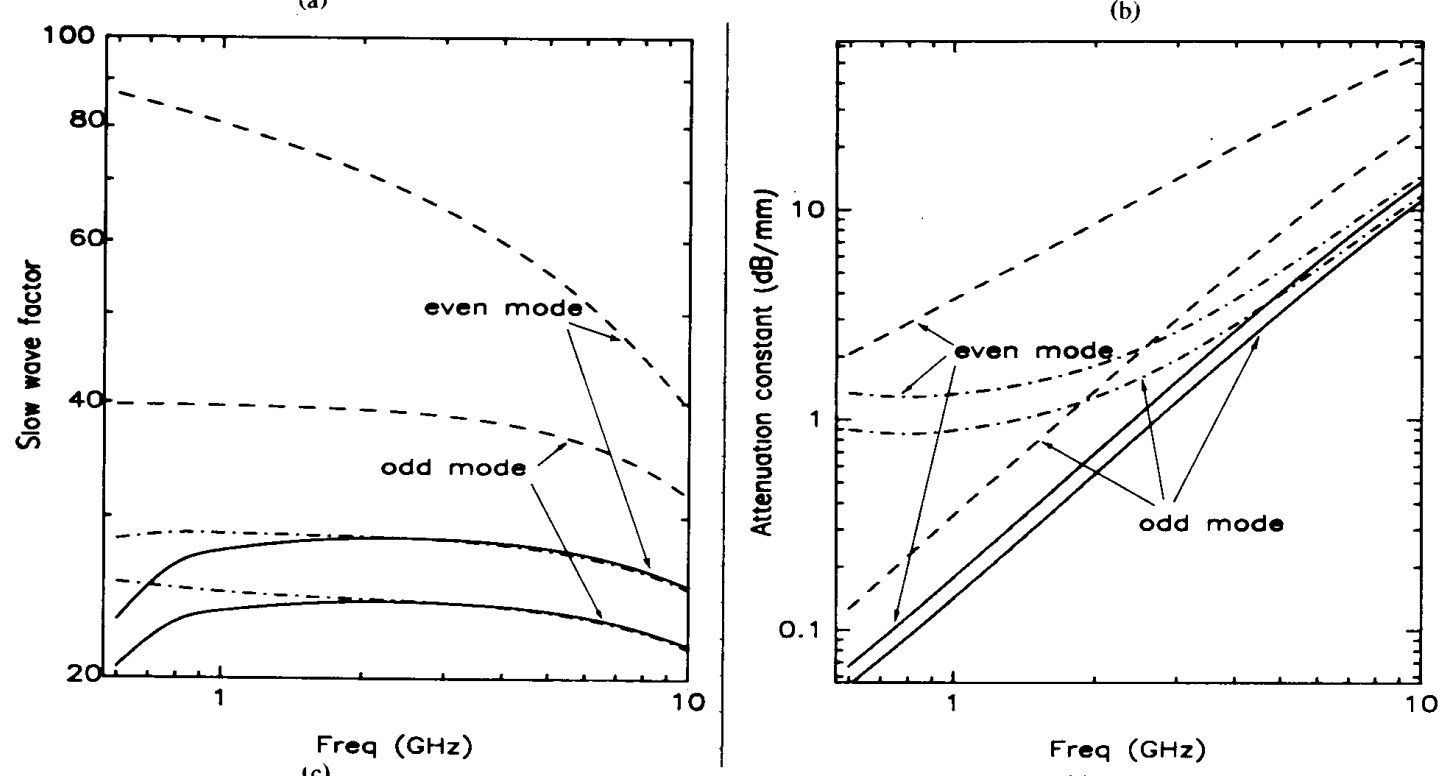

(d)

Fig. 7. For the configuration shown in Fig. 5, with $h_{1}=100 \mu \mathrm{m}, h_{2}=1 \mu \mathrm{m}, w=200 \mu \mathrm{m}, s=100 \mu \mathrm{m}, \epsilon_{r 1}=\epsilon_{r 2}=15.5$, $4 \pi M_{s}=0.2 \mathrm{kG}$, and $N=1$. (a) Slow-wave factor and (b) attenuation constant for a coupled microstrip demagnetized FM. (c) Slow-wave factor and (d) attenuation constant for a coupled microstrip demagnetized FMS: $\sigma=1(\Omega \cdot \mathrm{mm})^{-1}$. $(----)$ $A=100,(-\cdot-\cdot \cdot-) A=1,(-) A=0.01$

sor, where all the components can be different and com- with plex, leads to some differences with respect to the expression of these functions given in [15]. We have now

$$
\begin{aligned}
\frac{\tilde{g}_{i, i}(\alpha)}{\epsilon_{0}}= & j \alpha\left[\frac{\epsilon_{y x}^{i}-\epsilon_{x y}^{i}+\epsilon_{x y}^{i+1}-\epsilon_{y x}^{i+1}}{2}\right] \\
& +\epsilon_{y y}^{i} \alpha S_{i} \operatorname{coth}\left(\alpha S_{i} h_{i}\right) \\
& +\epsilon_{y y}^{i+1} \alpha S_{i+1} \operatorname{coth}\left(\alpha S_{i+1} h_{i+1}\right)
\end{aligned}
$$

$$
R_{i}=\frac{\epsilon_{x y}^{i}+\epsilon_{y x}^{i}}{2 \epsilon_{y y}^{i}} \quad \text { and } \quad S_{i}=\sqrt{\frac{\epsilon_{x x}^{i}}{\epsilon_{y y}^{i}}-R_{i}^{2}}
$$

Similarly,

$$
\frac{1}{\epsilon_{0}} \tilde{g}_{i, i+1}(\alpha)=-\epsilon_{y y}^{i+1} \alpha S_{i+1} \operatorname{csch}\left(\alpha S_{i+1} h_{i+1}\right) e^{j \alpha R_{i+1} h_{i+1}}
$$




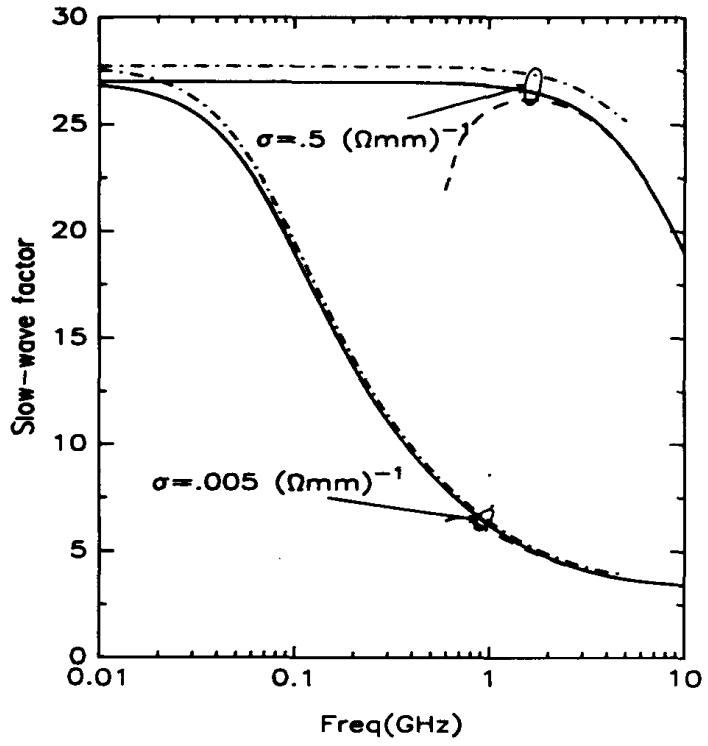

(a)

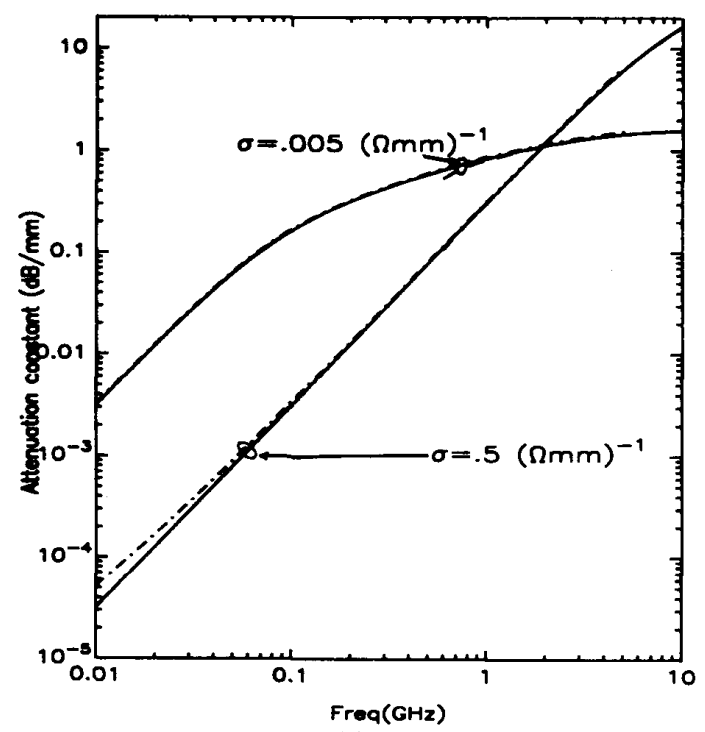

(b)

Fig. 8. (a) Slow-wave factor and (b) attenuation constant for different slow-wave microstrip configurations for two different values of conductivity. In Fig. $5 h_{1}=100 \mu \mathrm{m}, h_{2}=1 \mu \mathrm{m}, w=200 \mu \mathrm{m}, s \mapsto \infty$, and $\epsilon_{r 1}=\epsilon_{r 2}=15.5$. For demagnetized FMS, $4 \pi M_{s}=200$ G. $A=100$. $10^{-4}, N=1$. For saturated FMS, $H_{0}=2500$ Oe. $\Delta H=75$ Oe, $(\longrightarrow)$ MIS, (-- - ) demagnetized FMS, $(-\cdot-\cdot-\cdot-)$ saturated FMS.

and

$$
\frac{1}{\epsilon_{0}} \bar{g}_{i, i-1}(\alpha)=-\epsilon_{y y}^{i} \alpha S_{i} \operatorname{csch}\left(\alpha S_{i} h_{i}\right) e^{-j \alpha R_{i} h_{i}} .
$$

On the other hand, it is easy to check that the asymptotic behavior of the Green's function is

$$
\tilde{G}(\alpha) \mapsto \frac{K_{a s}}{\alpha} \quad \text { for } \alpha \mapsto \infty
$$

where

and

$$
K_{a s}=\frac{1}{j K+\epsilon_{y y}^{M} S_{m}+\epsilon_{y y}^{M+1} S_{M+1}}
$$

$$
K=\frac{\epsilon_{y x}^{M}+\epsilon_{x y}^{M+1}-\epsilon_{x y}^{M}-\epsilon_{y x}^{M+1}}{2}
$$

This asymptotic behavior for the Green's function is used in Appendix II in order to accelerate the convergence of integrals.

\section{APPENDIX II}

In order to obtain the value of the coefficients $\Gamma_{q m p n}$, it is necessary to calculate the following integrals:

$$
Z_{q m p n}=\int_{-\infty}^{\infty} J_{n}\left(\alpha \frac{w_{p}}{2}\right) \tilde{G}(\alpha) J_{m}\left(\alpha \frac{w_{q}}{2}\right) e^{j \alpha\left(c_{q}-c_{p}\right)} d \alpha .
$$

We choose as the asymptotic behavior of the integrand,

$$
J_{n}\left(\alpha \frac{w_{p}}{2}\right) \tilde{G}_{a s}(\alpha) J_{m}\left(\alpha \frac{w_{q}}{2}\right) e^{j \alpha\left(c_{q}-c_{p}\right)}
$$

with

$$
\tilde{G}_{a s}(\alpha)= \begin{cases}\frac{K_{a s}}{\frac{w_{q}}{2} \alpha \operatorname{coth}\left(\alpha \frac{w_{q}}{2}\right)} & \text { if } q=p \\ \frac{K_{a s}}{\alpha \operatorname{coth}(\alpha)} & \text { if } q \neq p .\end{cases}
$$

( $K_{a s}$ is defined in Appendix I.) Now we will separate the computation of $Z_{q m p n}$ into two parts in such a way that $Z_{q m p n}=Z_{q m p n}^{\prime}+Z_{q m p n}^{\prime \prime}$. The first term will be

$$
\begin{aligned}
Z_{q m p n}^{\prime}= & \int_{-\infty}^{\infty} J_{n}\left(\alpha \frac{w_{p}}{2}\right)\left[\tilde{G}(\alpha)-\tilde{G}_{a s}(\alpha)\right] \\
& \cdot J_{m}\left(\alpha \frac{w_{q}}{2}\right) e^{j \alpha\left(c_{q}-c_{p}\right)} d \alpha .
\end{aligned}
$$

It will converge fast because the integrand is practically zero when $|\alpha| \geqslant 4$. Then, by means of any numerical integration method, the computation time of this term is very short. The second term is

$$
Z_{q m p n}^{\prime \prime}=\int_{-\infty}^{\infty} J_{n}\left(\alpha w_{p} / 2\right) \tilde{G}_{a s}(\alpha) J_{m}\left(\alpha w_{q} / 2\right) e^{j \alpha\left(c_{q}-c_{p}\right)} d \alpha,
$$

and two cases are considered. The first is where $q=p$ :

$$
Z_{q m p n}^{\prime \prime}=\int_{-\infty}^{\infty} J_{n}\left(\alpha \frac{w_{q}}{2}\right) \frac{K_{a s}}{\frac{w_{q}}{2} \alpha \operatorname{coth}\left(\alpha \frac{w_{q}}{2}\right)} J_{m}\left(\alpha \frac{w_{q}}{2}\right) d \alpha .
$$

This integral can be normalized and then it will not depend on the dimension of the structure, so integrals of this kind are computed just once and then tabulated. The second case is where $q \neq p$ :

$Z_{q m p n}^{\prime \prime}=K_{a s} \int_{-\infty}^{\infty} \frac{J_{n}\left(\alpha \frac{w_{p}}{2}\right) J_{m}\left(\alpha \frac{w_{q}}{2}\right) e^{j \alpha\left(c_{q}-c_{p}\right)} \sinh (\alpha)}{\alpha \cosh (\alpha)} d \alpha$. 
These coefficients are calculated by applying residue calculus and choosing the appropriate integration path in the complex field. After some manipulations,

$$
\frac{Z_{q m p n}^{\prime \prime}}{K_{a s}}= \begin{cases}2 \pi j^{m+n} \sum_{k=1}^{\infty} I_{m}\left(a_{k} \frac{w_{q}}{2}\right) I_{n}\left(a_{k} \frac{w_{p}}{2}\right) \frac{e^{-a_{k}\left(c_{q}-c_{p}\right)}}{a_{k}} & \text { if } q>p \\ 2 \pi(-j)^{m+n} \sum_{k=1}^{\infty} I_{m}\left(a_{k} \frac{w_{q}}{2}\right) I_{n}\left(a_{k} \frac{w_{p}}{2}\right) \frac{e^{a_{k}\left(c_{q}-c_{p}\right)}}{a_{k}} & \text { if } q<p .\end{cases}
$$

Here $a_{k}=((2 k-1) / 2) \pi$ and $I_{m n}$ is the modified Bessel function of order $m$.

These series converge very rapidly. In practice, just four or five terms are necessary for a very good approximation (five to seven significant digits).

\section{REFERENCES}

[1] P. Kennis and L. Faucon, "Rigorous analysis of planar MIS transmission lines," Electron. Lett., vol. 17, no. 13, pp. 454-456, June 1981.

[2] T. Mu, H. Ogawa, and T. Itoh, "Characteristics of multiconductor. asymmetric, slow-wave microstrip transmission lines," IEEE Trans. Microwace Theory Tech., vol. MTT-34, pp. 1471-1477, Dec. 1986.

[3] Y. R. Kwon, V. M. Hietala, and K. S. Champlin, "Quasi-TEM analysis of "slow-wave" mode propagation on coplanar microstructure MIS transmission lines" IEEE Trans. Microware Theory Tech., vol. MTT-35, pp. 545-551, June 1987.

[4] H. Ogawa and T. Itoh, "Slow-wave characteristics of ferromagnetic semiconductor microstrip line," IEEE Trans. Microwale Theory Tech., vol. MTT-34, pp. 1478-1482, Dec. 1986.

[5] T. Kitazawa, "Variational method for multiconductor coupled striplines with stratified anisotropic media," IEEE Trans. Mi crowace Theory Tech., vol. 37, pp. 484-491, Nov. 1979.

[6] R. Marques and M. Horno, "Propagation of quasi-static modes in anisotropic transmission lines: Application to MIC lines," IEEE Trans. Microwave Theon Tech., vol. MTT-33, pp. 927-932. Oct. 1985.

[7] I. V. Lindell, "On the quasi-TEM modes in inhomogeneous multiconductor transmission lines," IEEE Trans. Microwace Theory Tech., vol. MTT-29, pp. 812-817, Aug. 1981.

[8] J. J. Green and F. Sandy, "A catalog of low power loss parameter and high power thresholds for partially magnetized ferrites," IEEE Trans. Microwace Theory Tech., vol. MTT-22, pp. 645-651, June 1974.

[9] R. A. Pucel and D. J. Masse, "Microstrip propagation on magnetic substrates-Part I: Design Theory," IEEE Trans. Microwace The ory Tech., vol. MTT-20, pp. 304-308, May 1972.

[10] J. R. Mautz, R. F. Harington, and C. G. Hsu, "The inductance matrix of a multiconductor transmission line in a multiple mag. netic media," IEEE Trans. Microwate Theory Tech., vol. 36, pp. 1293-1295, Aug. 1988.

[11] R. F. Harrington and C. Wei, "Losses on multiconductor transmission lines in multilayered dielectric media," IEEE Trans. Microwace Theory Tech., vol. MTT-32, pp. 705-710, July 1984.

[12] F. Medina and M. Horno, "Upper and lower bounds on mode capacitances for a large class of anisotropic multilayered microstrip-like transmission lines," Proc. Inst. Elec. Eng. (Microwates, Optics \& Antennas), vol. 123, no. 3, pp. 157-163, Jun 1985.

[13] E. J. Denlinger, "A frequency dependent solution for microstrip transmission lines," IEEE Trans. Microwace Theory Tech., vol. MTT-19, pp. 30-39, Jan. 1979.

[14] L. Young and H. Sobol, Eds., Adrances in Microwate, vol. 8. New York: Academic Press, 1974.

[15] F. Medina, "Study of generalised shielded planar transmission lines on anisotropic dielectric" (in Spanish), Ph.D. thesis, Electronic and Electromagnetism Department, Univ. of Seville, Spain, 1987.

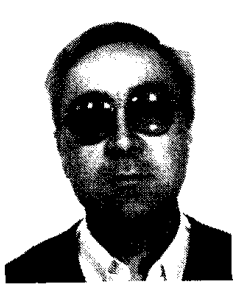

Manuel Horno (M'75) was born in Torre del Campo, Jaén, Spain. He received the degree of Licenciado in physics in June 1969 and the degree of Doctor en Ciencias in physics in January 1972, both from the University of Sevilla, Spain.

Since October 1969 he has been with the Department of Electricity and Electronics at the University of Sevilla, where he became an Assistant Professor in 1970, an Associate Professor in 1975, and a Professor in 1986. His main fields of interest include boundary value problems in electromagnetic theory, wave propagation through anisotropic media, and microwave integrated circuits. He is presently engaged in the analysis of planar transmission lines embedded in anisotropic materials, multiconductor transmission lines, and planar slow-wave structures.

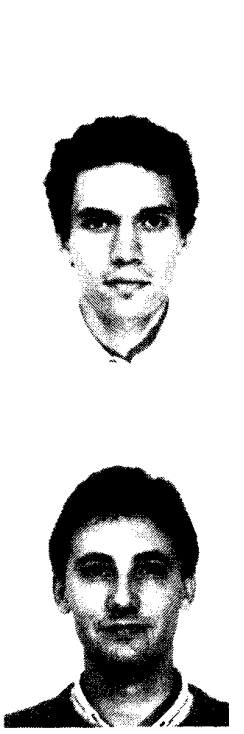

Francisco L. Mesa was born in Cádiz, Spain, in April 1965. He received the degree of Licenciado in physics in June 1989 from the University of Sevilla, Spain. He is currently in a Ph.D program in microwaves with a scholarship from the Spanish Government. His research interests focus on planar lines on general anisotropic materials.

里

Francisco Medina was born in Puerto Real, Cádiz, Spain, on November 9, 1960. He received the Licenciado degree in September 1983 and the doctor degree in September 1987, both in physics, from the University of Sevilla, Spain.

$\mathrm{He}$ is currently Assistant Professor of Electricity and Magnetism in the Department of Electronics and Electromagnetism, University of Sevilla. His research deals mainly with numerical methods for planar structures and multiconductor lines.

出

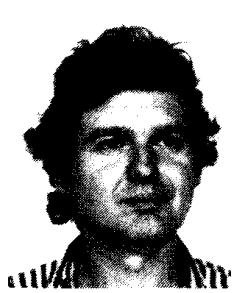

Ricardo Marqués was born in San Fernando, Cádiz, Spain. He received the degree of Licenciado in physics in June 1983 and the degree of doctor in physics in July 1987, both from the University of Sevilla.

Since January 1984, he has been with the Department of Electronics and Electromag netism at the University of Sevilla, where he is currently Assistant Professor of Electricity and Magnetism. His main fields of interest include MIC devices design, wave propagation in anisotropic media, and electromagnetic theory. 